\title{
Characterization of Calcium Oxalate Biominerals in Pereskia Species (Cactaceae)
}

\author{
Paula V. Monje and Enrique J. Baran* \\ Centro de Química Inorgánica (CEQUINOR, CONICET/UNLP), Facultad de Ciencias \\ Exactas, Universidad Nacional de La Plata, C. Correo 962, 1900 La Plata, Argentina. \\ E-mail: baran@quimica.unlp.edu.ar \\ * Author for correspondence and reprint requests \\ Z. Naturforsch. 64c, 763-766 (2009); received June 29, 2009 \\ Calcium oxalate druses were isolated from the stems and leaves of six Pereskioideae \\ family members and investigated by infrared spectroscopy, showing that in all samples the \\ biomineral was present in the form of whewellite, $\mathrm{CaC}_{2} \mathrm{O}_{4} \cdot \mathrm{H}_{2} \mathrm{O}$. As Pereskia is thought to \\ represent the "ancestral" condition of the leafless stem-succulent cacti, these results suggest \\ that the biomineralization of calcium oxalate in Cactaceae represents a primitive charac- \\ teristic of the group and also support a close genetic relationship between Pereskia and \\ Opuntia.
}

Key words: Pereskia, Biominerals, Whewellite, Infrared Spectroscopy

\section{Introduction}

Crystalline oxalates are widely distributed in nature and have been observed in rocks, soil, and among a variety of living organisms, including plants and animals (Baran and Monje, 2008). The most widely distributed biomineral of this type is calcium oxalate, which is especially common in the plant kingdom (Arnott, 1982; Khan, 1995; Monje and Baran, 2004a; Baran and Monje, 2008). The presence of other metal oxalates is extremely rare in biological systems, although the presence of crystalline magnesium, manganese, copper, and ammonium oxalates in different forms of life has been reported, whereas soluble forms of sodium and potassium oxalates are widely distributed in plants and fungi (Baran and Monje, 2008).

Crystalline calcium oxalates have been found in two different hydration states in plants, either as the monoclinic monohydrate (whewellite, $\mathrm{CaC}_{2} \mathrm{O}_{4}$ - $\mathrm{H}_{2} \mathrm{O}$ ) or as the orthorhombic dihydrate (weddellite, $\mathrm{CaC}_{2} \mathrm{O}_{4} \cdot 2 \mathrm{H}_{2} \mathrm{O}$ ). Whewellite is the most stable form from the thermodynamic point of view (Monje and Baran, 2004a; Baran and Monje, 2008). However, the morphology (druses, raphids, solitary crystals, crystal sand) and the hydration state of calcium oxalates in plants differ significantly among different groups.

Because higher plants incorporate calcium in excess to the cellular requirements and most of them, unlike animals, do not have well-developed excretory systems to eliminate such excess, they modulate the difference between the natural abundance of calcium and the very low intracellular levels required by tightly controlling the distribution of calcium and its compartmentation within cells. In this context, with a solubility product of $1.3 \cdot 10^{-9} \mathrm{~mol}^{2} \mathrm{l}^{-2}$ in water, calcium oxalate effectively sequesters calcium and renders itself metabolically and osmotically inactive for plant cells. Therefore, many plants accumulate crystalline calcium oxalate in response to the presence of an excess of calcium (Webb, 1999; Baran and Monje, 2008).

On the other hand, a number of recent studies indicate that these calcium oxalate crystals do not form an inert, non-retrievable deposit of calcium, but can act as a sort of reservoir to which plants can recur in cases of calcium deficiency. Apart from this primary function as a calcium regulator, the great variety of crystal shapes and sizes, as well as their localization in different plant tissues, suggests various other functions for calcium oxalates which might be evolved secondarily. Some of these functions include mechanical support, gravity perception, intracellular $\mathrm{pH}$ regulation and ion balance, detoxification and even light gathering and reflection (Nakata, 2003; Baran and Monje, 2008).

The Cactaceae family is a well-known lineage of stem-succulent plants that are widely recog- 
nized for their specialized adaptation to drought. These include an extensive and shallow root system with dynamic hydraulic properties that allow for maximum water uptake following rain, a succulent pith and cortex with high capacitance for water storage, and a long-lived stem cortical tissue system that has replaced leaves as the primary photosynthetic organ. These anatomical and histological adaptations come along with the acquisition of crassulacean acid metabolism (CAM photosynthesis), which minimizes the transpirational water loss by allowing the stomata to open during the night (Edwards and Diaz, 2006; Edwards and Donoghue, 2006). Cactaceae species are also unique in their ability to biomineralize calcium oxalate (Rivera and Smith, 1979; Monje and Baran, 2002; Hartl et al., 2007), and the content of the biomineral can reach up to $80-90 \%$ of the plant's dry weight (Monje and Baran, 2004a). However, the connection between calcium oxalate biomineralization and water stress adaptation remains obscure.

The Pereskia genus (subfamily: Pereskioideae, family: Cactaceae) consists of species of leafy shrubs and trees and is the only cactus group that has persistent non-succulent stems and leaves. It has long been considered as the best living representation of the "ancestral cactus" (Edwards et al., 2005; Edwards and Donoghue, 2006; Metzing and Kiesling, 2008). As opposed to succulent cacti, most species of Pereskioideae grow in tropical, humid environments.

We have extensively studied the biominerals present in the two main succulent subfamilies of cacti, Cereoideae and Opuntioideae, and have found the presence of calcium oxalate (Monje and Baran, 1996, 1997, 2002, 2004b), magnesium oxalate (Monje and Baran, 2005), and silicon dioxide (Monje and Baran, 2000, 2004b). We have now investigated the presence of calcium oxalates in different species of Pereskia, the non-succulent "relictual cacti", in order to investigate the possible relationship between calcium oxalate biomineralization and adaptation of cacti drought.

\section{Material and Methods}

\section{Plant material}

The plant material was provided by the South Florida Cactus and Succulent Society (Miami, Florida). The stems and leaves of six representative species of Pereskia were harvested from healthy and well-hydrated specimens growing under normal greenhouse conditions, regardless of the season and other environmental conditions.

\section{Crystal isolation and purification}

Crystal druses were isolated from fresh plant tissues. Plant stems and leaves were carefully washed with abundant distilled water to remove any possible external contamination, cut with a razor blade into thin tissue sections and immersed in a $6 \%$ sodium hypochlorite solution for $48 \mathrm{~h}$. The tissue was then mechanically dissociated by passing it repeatedly through a plastic transfer pipette, filtered through gauze, and allowed to decant for $30 \mathrm{~min}$. The pellet containing crystalline products was suspended in absolute ethanol, and the crystals were manually collected by inspection through a dissecting light microscope. The isolated crystalline material was repeatedly washed with ethanol until the tissue debris was no longer evident. This procedure renders crystalline samples consisting mainly of intact druses. The purified crystal samples were finally dried under a nitrogen flow before spectroscopic analysis.

\section{Synthesis of $\mathrm{CaC}_{2} \mathrm{O}_{4} \cdot \mathrm{H}_{2} \mathrm{O}$ samples}

For comparative purposes we have prepared high-purity crystalline $\mathrm{CaC}_{2} \mathrm{O}_{4} \cdot \mathrm{H}_{2} \mathrm{O}$ samples. They were prepared by simultaneous dropping of highly diluted sodium oxalate and calcium chloride solutions in a great volume of distilled water, maintained at $70^{\circ} \mathrm{C}$, as described by Grases et al. (1990).

\section{Infrared spectroscopy}

The IR spectra were obtained by means of a Bruker IFS 66 spectrophotometer in the spectral range between 4000 and $400 \mathrm{~cm}^{-1}$ using the $\mathrm{KBr}$ pellet technique ( $4 \mathrm{mg}$ of the powdered sample dispersed in $100 \mathrm{mg}$ of $\mathrm{KBr}$ ).

\section{Results and Discussion}

Infrared spectroscopy is a powerful tool for the investigation of the chemical composition of plant material (Baran, 2005); we have applied this methodology successfully in the investigation of plant biominerals (Monje and Baran, 1996, 1997, 2000, 2002, 2004b, 2005; Baran and Rolleri, 2009). In the present study, we have used this sensitive spectroscopic technique for the first time to analyze the 
composition of crystalline material isolated from the stems and leaves of six different members of the Pereskiodeae subfamily (Pereskia aculeata, $P$. bleo, $P$. portulacifolia, P. nicoyana, $P$. quisqueyana, and Pereskiopsis sp.); all species showed the presence of highly abundant druses in tissues of the stems and leaves.

The infrared spectra of biominerals present in the purified druses of Pereskia species showed clearly that the crystals consisted of highly pure whewellite $\left(\mathrm{CaC}_{2} \mathrm{O}_{4} \cdot \mathrm{H}_{2} \mathrm{O}\right)$. This was a consistent finding regardless of the species analyzed and their localization in the plant body. Fig. 1 shows a comparison of the infrared spectra of a pure synthetic sample of $\mathrm{CaC}_{2} \mathrm{O}_{4} \cdot \mathrm{H}_{2} \mathrm{O}$ a representative crystalline sample of Pereskia (P. bleo), and a crystalline sample isolated from an Opuntioideae family member (Tephrocactus articulatus), that was identified as whewellite in a previous study (Monje and Baran, 2002). As it can be seen the three spectra are identical, indicating that whew-

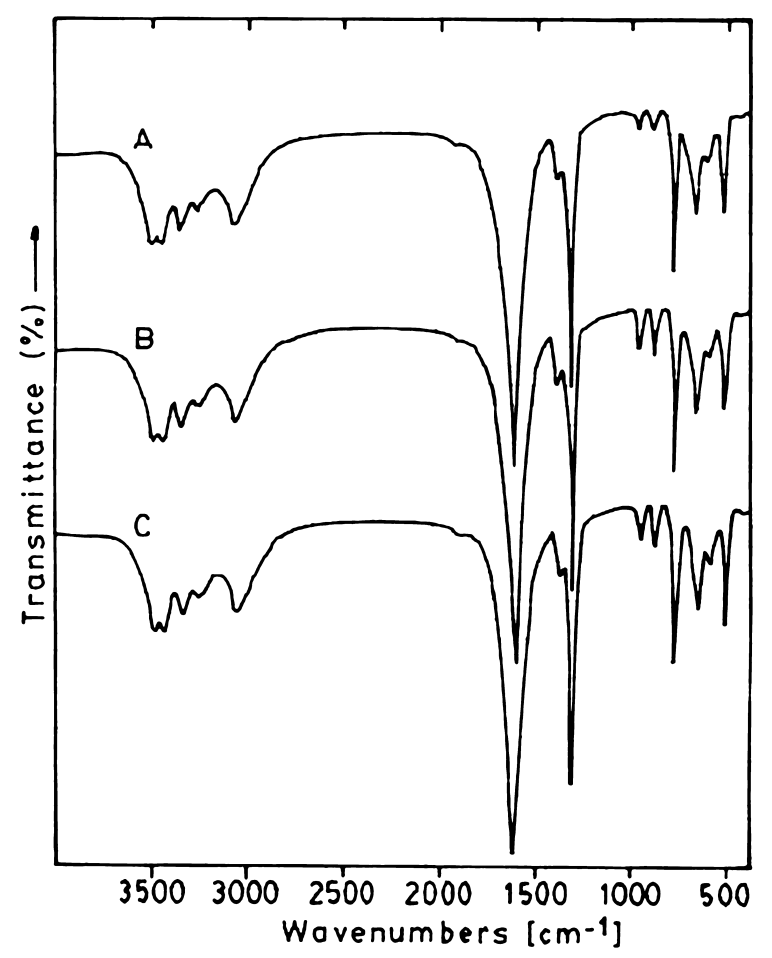

Fig. 1. FTIR-spectra of (A) a pure sample of synthetic $\mathrm{CaC}_{2} \mathrm{O}_{4} \cdot \mathrm{H}_{2} \mathrm{O}$, (B) druses of Pereskia bleo and (C) druses of Tephrocactus articulatus. ellite is the main biomineral present in the druses of Pereskioideae members. Four other species of this family ( $P$. grandifolia, $P$. sacharosa, $P$. stenantha, $P$. zinniflora) recently, investigated by $\mathrm{X}$-ray diffractometry, also show the presence of whewellite (Hartl et al., 2007).

Our previous investigations on biominerals present in the Cactaceae family have shown that Opuntioideae family members biomineralize calcium oxalate as whewellite, $\mathrm{CaC}_{2} \mathrm{O}_{4} \cdot \mathrm{H}_{2} \mathrm{O}$, whereas Cereoideae members biomineralize calcium oxalate as weddellite, $\mathrm{CaC}_{2} \mathrm{O}_{4} \cdot 2 \mathrm{H}_{2} \mathrm{O}$, suggesting a definite but different genetic control over the biomineralization process in the two most representative groups of the succulent Cactaceae (Monje and Baran 2002, 2004a). This conclusion was confirmed by a recent X-ray diffraction study of the total crystalline material present in a large number of Cactaceae species (Hartl et al., 2007). Even though the simultaneous presence of the two hydration states of calcium oxalate in a single taxon seems to be rare in angiosperms (Arnott, 1982), Hartl et al. (2007) showed that certain Cactaceae species display the concurrent presence of weddellite and whewellite. One of the few known examples in which the presence of both forms of calcium oxalate has been unambiguously demonstrated is Opuntia ficus indica (Malainine et al., 2003).

Overall, the results of our study confirmed a close genetic relationship between Pereskia and Opuntia (Metzing and Kiesling, 2008), as both groups biomineralize calcium oxalate as whewellite druses, which were also highly similar in size and morphology. Therefore, we conclude that whewellite must have been the ancestral biomineral in the Cactaceae lineage and that weddellite must have evolved after branching of the Cereus as a separate group. Besides, the observation of whewellite druses in all species of Pereskia suggests that the biomineralization of calcium oxalate in Cactaceae represents a primitive characteristic of the family, most likely acquired before adaptation to water stress conditions. Whereas primitive cacti deposit calcium oxalate in the form of the thermodynamically most stable monohydrate, in the course of evolution certain lines of Cactaceae perhaps conserved calcium oxalate mainly in the form of the metastable and convertible dihydrate. 


\section{Acknowledgements}

This work was supported by Universidad Nacional de La Plata (UNLP) and Consejo Nacional de Investigaciones Científicas y Técnicas de

Arnott H. J. (1982), Three systems of biomineralization in plants with comments on the associated organic matrix. In: Biological Mineralization and Demineralization. (Nancollas G. H., ed.). Springer, Berlin, pp. 199-218.

Baran E. J. (2005), Applications of vibrational spectroscopy to the investigation of plant material. In: Advances in Plant Physiology, Vol. 8 (Hemantaranjan H., ed.). Scientific Publishers, Jodhpur, pp. 365-392.

Baran E. J. and Monje P. V. (2008), Oxalate biominerals. In: Metal Ions in Life Sciences, Vol. 4 (Sigel A., Sigel H., and Sigel R. K. O., eds.). J. Wiley, Chichester, pp. 219-254.

Baran E. J. and Rolleri C. H. (2009), IR-spectroscopic characterization of biominerals in the marattiaceaeus ferns Rev. Bras. Bot. (in press).

Edwards E. J. and Diaz M. (2006), Ecological physiology of Pereskia guamacho, a cactus with leaves. Plant Cell Environm. 29, 247-256.

Edwards E. J. and Donoghue M. J. (2006), Pereskia and the origin of the cactus life-form. Am. Nat. 167, $777-793$.

Edwards E. J., Nyffeler R., and Donoghue M. J. (2005), Basal cactus phylogeny: implication of Pereskia (Cactaceae) paraphyl for the transition to the cactus life form. Am. J. Bot. 92, 1177-1188.

Grases F., Millan A., and Conte A. (1990), Production of calcium oxalate monohydrate, dihydrate or trihydrate. Urol. Res. 18, 17-20.

Hartl W. P., Klapper H., Barbier B., Ensikat H. J., Dronskowski R., Müller P., Ostendorp G., Tye A., Bauer R., and Barthlott W. (2007), Diversity of calcium oxalate crystals in Cactaceae. Can. J. Bot. 85, 501-517.

Khan S. R. (ed.) (1995), Calcium Oxalate in Biological Systems. CRC-Press, Boca Raton.

Malainine M. E., Dufresne A., Dupeyre D., Vignon M.R., and Mahrouz M. (2003), First evidence for the la República Argentina (CONICET). E. J. B. is a member of the Research Career of this organism.

presence of weddellite crystallites in Opuntia ficus indica parenchyma. Z. Naturforsch. 58c, 812-816.

Metzing D. and Kiesling R. (2008). The study of cactus evolution: The pre-DNA era. Haseltonia 14, 6-25.

Monje P. V. and Baran E. J. (1996), On the formation of weddellite in Chamaecereus silvestrii, a Cactaceae species from northern Argentina. Z. Naturforsch. 51c, 426-428.

Monje P. V. and Baran E. J. (1997), On the formation of whewellite in the Cactaceae species Opuntia microdasys. Z. Naturforsch. 52c, 267-269.

Monje P. V. and Baran E. J. (2000), First evidences of the bioaccumulation of $\alpha$-quartz in a Cactaceae. J. Plant Physiol. 157, 457-460.

Monje P. V. and Baran E. J. (2002), Characterization of calcium oxalates generated as biominerals in cacti. Plant Physiol. 128, 707-713.

Monje P. V. and Baran E. J. (2004a), Plant biomineralization. In: Advances in Plant Physiology, Vol. 7 (Hemantaranjan A., ed.), Scientific Publishers, Jodhpur, pp. 395-410.

Monje P. V. and Baran E. J. (2004b), Complex biomineralization pattern in Cactaceae. J. Plant Physiol. 161, $121-123$.

Monje P. V. and Baran E. J. (2005), Evidence of the formation of glushinskite as a biomineral in a Cactaceae species. Phytochemistry 66, 611-614.

Nakata P. A. (2003), Advances in our understanding of calcium oxalate crystal formation and function in plants. Plant Sci. 164, 901-909.

Rivera E. R. and Smith B. N. (1979), Crystal morphology and ${ }^{13}$ Carbon $/{ }^{12}$ Carbon composition of solid oxalate in cacti. Plant Physiol. 64, 966-970.

Webb M. A. (1999), Cell-mediated crystallization of calcium oxalate in plants. Plant Cell 11, 751-761. 